\title{
Passive control of resonances by nonlinear absorbers
}

\author{
J.W. Shao ${ }^{1,2, a}$ and B. Cochelin ${ }^{1,2}$ \\ 1 Laboratory of Mechanics and Acoustics (LMA), chemin Joseph-Aiguier, 13402, MARSEILLE \\ 2 Ecole Centrale Marseille (ECM), rue Frederic Joliot-Curie, 13451, MARSEILLE
}

\begin{abstract}
A new passive technique for reducing vibration and noise using purely nonlinear absorbers is studied. Compared to classical passive systems (Frahm absorbers, Helmholtz resonator), a single nonlinear absorber works in a limited range of forcing but in a wide band of frequency. In this paper, a simple system with two degrees of freedom is used to analyze the beginning of the "Targeted Energy Transfer" TET phenomenon and to define the desired working zone for Nonlinear Energy Sink (NES). An analytical formula of the level of forcing for the beginning of TET is established by a simplified approach.
\end{abstract}

\section{Introduction}

The classical linear systems (such as the Frahm absorber and the Helmholtz resonator) are often used for passive control of vibration and noise. However, these systems are only effective in a very limited frequency band. A new passive technique for reducing noise and vibration is to use purely nonlinear absorbers working on the principle of "Targeted Energy Transfer" (TET). The concept of TET was first proposed by Vakakis and Gendelman [1,2], in 2001. We often speak of Nonlinear Energy Sink (NES) for a purely nonlinear absorber. The first experimental demonstration of the phenomenon of TET was published in 2005 [3]. Since then, several theoretical and experimental studies have been made in view of applications in the field of mechanical vibration. Recently the ideas of NES found successful implementations not only in vibration attenuation of the unwanted mechanical vibrations, but also in the acoustics field where the aforementioned methodology is used for a passive sound control in low frequency domain. Cochelin et al. [4] and Bellet et al. [5] have conducted a comprehensive study on the use of a thin viscoelastic membrane for the design of a NES in acoustics. They experimentally investigate the TET between the membrane and the acoustic medium for both free and forced oscillations. Mariani et al. [6] conducted a complementary study to show that a loudspeaker working outside its linear regime can also be an effecient nonlinear absorber.

In this paper, we analyze the TET phenomenon by using a simple system with two degrees of freedom, which is constituted by a primary linear system and an attached NES. We concentrate on the case where a harmonic source excitation is applied to the primary linear system and we look for the reduction of the resonance peak by mean of the so-called "Strongly Modulated Response" (SMR). This particular type of response is characterized by a repetitive occurence of the TET which permits to limit the response amplitude of the resonance peak. Finding the threshold value of the forcing level beyong which SMR begins has been recently adressed in [7] and [8] by means of theo-

\footnotetext{
a e-mail: shao@lma.cnrs-mrs.fr
}

retical analysis with multiple time scale and complexification method. The results are however hardly transposable for the specific grounded system used in [5]. We will here follow a simplified approach by determining the nonlinear modes and the periodic responses of the system and by using a harmonic-based numerical method to analyze their stability. Looking for the first destabilization of the resonance peak of the periodic response and the appearance of the additional branches of periodic regimes at a higher amplitude of the forcing, we analyze the beginning of the TET phenomenon and finally define "the desired working zone" for the NES. An analytical formula of the level of forcing for the beginning of TET ("the threshold for the beginning of TET") is established by a simplified approach. The intended application of this study is to treat the low frequency noise attenuation, where no effective disspative mechanism exists.

\section{Targeted energy transfer under harmonic forcing}

\subsection{Description of the model}

We consider a simple system with two degrees of freedom, which is constituted by a linear system with one degree of freedom (the first oscillator, or the primary system) and a NES (the second oscillator, or nonlinear absorber). The first oscillator is a classical linear system mass-springdamping and the second system is comprised by a mass, a purely nonlinear spring with cubic stiffness and a viscous damper. The two oscillators are coupled by a linear spring with weak stiffness. A periodic forcing $f(t)$ is applied to the primary oscillator.

After defining a dimensionless time $\tau=t \omega_{1}$, where $\omega_{1}=\sqrt{k_{1} / m_{1}}$, the equations of the system (Fig. 1) are represented in the following dimensionless form:

$$
\begin{aligned}
& \ddot{u}_{1}+\mu_{1} \dot{u}_{1}+u_{1}+\beta\left(u_{1}-u_{a}\right)=F(t) \\
& \gamma \ddot{u}_{a}+\mu_{a} \dot{u}_{a}+\alpha_{a} u_{a}^{3}+\beta\left(u_{a}-u_{1}\right)=0
\end{aligned}
$$

where $\gamma=m_{a} / m_{1}$ is the ratio of two masses, $\beta=k_{c} / k_{1}$ the weak coefficient of coupling, $\mu_{1}=c_{1} / \sqrt{k_{1} m_{1}}, \mu_{a}=$ 


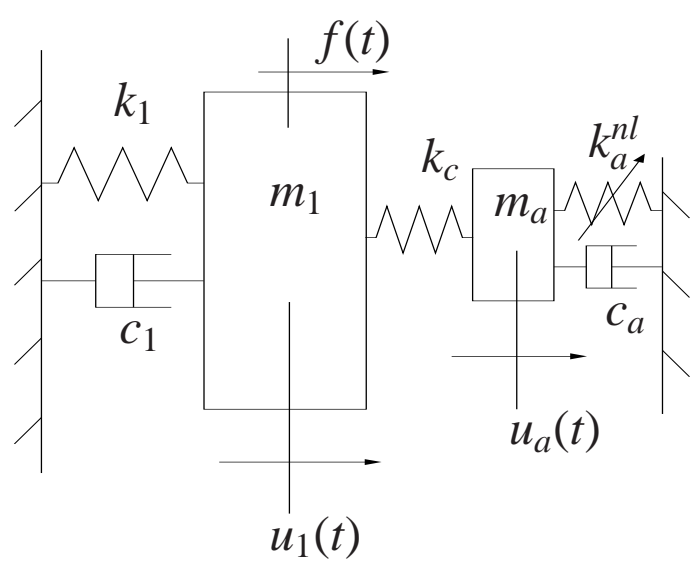

Fig. 1. The system with two degrees of freedom for TET

$c_{a} / \sqrt{k_{1} m_{1}}$ the respective damping ratio of the primary system and NES and $\alpha_{a}=k_{a}^{n l} / k_{1}$ the coefficient of the cubic stiffness. For illustration, we choose the parameters $\gamma=1$, $\beta=0.15, \mu_{1}=0.01, \mu_{a}=0.18, \alpha_{a}=1$.

\subsection{Nonlinear normal modes}

It is important to look at the nonlinear normal modes of the system because the TET phenomenon is caused by a 1:1 resonance capture [2]. In order to analyze the two nonlinear normal modes, we remove the force $F$ and all the dampings in the system (1). A simplified analysis of the nonlinear normal modes is done by using a harmonic balance method (HBM) with a single term, as follows $u_{1}(t)=$ $u_{1 c} \cos (\omega t)$ and $u_{a}(t)=u_{a c} \cos (\omega t)$. Then, the following two algebraic equations are obtained:

$$
\begin{aligned}
& \left(1+\beta-\omega^{2}\right) u_{1 c}-\beta u_{a c}=0 \\
& -\beta u_{1 c}+\left(\beta-\gamma \omega^{2}\right) u_{a c}+\frac{3}{4} \alpha_{a} u_{a c}^{3}=0
\end{aligned}
$$

The nonlinear normal modes are shown in Fig. 2. For the first nonlinear mode, $u_{1}(t)$ and $u_{a}(t)$ oscillate in phase, while for the second nonlinear mode, $u_{1}(t)$ and $u_{a}(t)$ oscillate out of phase.

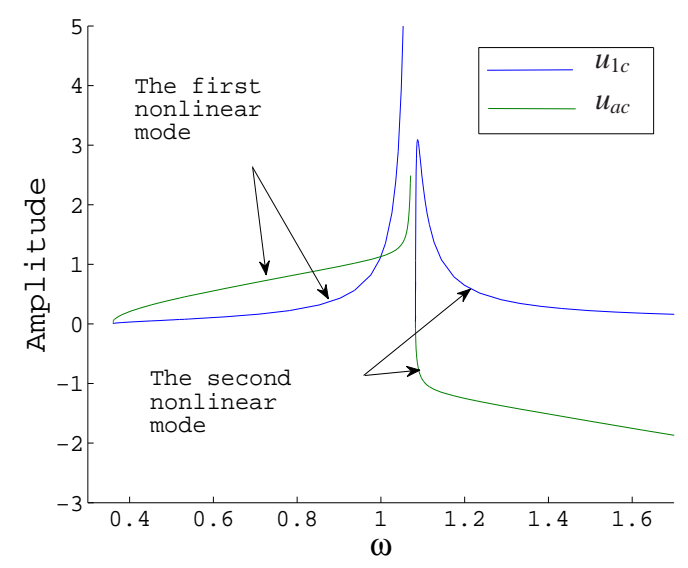

Fig. 2. Nonlinear normal modes of the system.

\subsection{Response to periodic forcing}

We now look for the periodic forced responses of the system (1) when the forcing term $F(t)$ is harmonic with a single frequency $\omega$. We restrict the analysis to $1: 1$ fundamental resonance by applying the HBM with a single harmonic term. Since the nonlinear term of (1) is $u_{a}^{3}$, we set $\dot{u}_{a}(0)=0$ and take the force $F(t)=F_{1 c} \cos (\omega t)+F_{1 s} \sin (\omega t)$ and the displacements $u_{1}(t)=u_{1 c} \cos (\omega t)+u_{1 s} \sin (\omega t)$ and $u_{a}(t)=u_{a c} \cos (\omega t)$. Introducing into (1) and neglecting the higher harmonics in $3 \omega$, we obtain the following four algebraic equations for the six unknows $F_{1 c}, F_{1 s}, u_{1 c}, u_{1 s}, u_{a c}$ and $\omega$ :

$$
\begin{aligned}
& \left(1+\beta-\omega^{2}\right) u_{1 c}+\mu_{1} \omega u_{1 s}-\beta u_{a c}=F_{1 c} \\
& \left(1+\beta-\omega^{2}\right) u_{1 s}-\mu_{1} \omega u_{1 c}=F_{1 s} \\
& \frac{3}{4} \alpha_{a} u_{a c}^{3}+\left(\beta-\gamma \omega^{2}\right) u_{a c}-\beta u_{1 c}=0 \\
& \mu_{a} \omega u_{a c}+\beta u_{1 s}=0
\end{aligned}
$$

We define $F_{1}=\sqrt{F_{1 c}^{2}+F_{1 s}^{2}}$ and $u_{1}=\sqrt{u_{1 c}^{2}+u_{1 s}^{2}}$. In the algebraic equation (3), there is only one nonlinear term $u_{a c}^{3}$. We take the amplitude $u_{a c}$ and the frequency $\omega$ as the master parameters, then closed form expressions can be easily obtained for the response surfaces. A set of periodic responses of the system under forcing regime is shown in Fig. 3(b) and Fig. 3(d). We have also calculated two nonlinear modes shown in Fig. 3(a) and Fig. 3(c). Here, we represent the absolute values of the amplitudes of $u_{1}(t)$ and $u_{a}(t)$ of two nonlinear modes in order to see the periodic forced responses. We can see that the curves of forced responses remain in the vicinity of the nonlinear modes. In Figure 3(d), when the forcing level remains under a certain range, the resonance peaks of $u_{1}(t)$ are clipped and limited just like a plateau. When the forcing level exceeds that range, the additional branches of periodic regimes with rather high amplitudes appear, which look like some "closed loops" in Figure 3(d). Here, we observe that the value of the plateau corresponds with the maximum point of the second nonlinear mode of $u_{1}(t)$ in Figure 3(c). In the following section, we will show that from this point, the second nonlinear mode of $u_{1}(t)$ becomes instable. Regarding the forced responses, when the resonance peak reaches this point, the periodic forced solutions will also destabilize in favor of SMRs.

\section{Analysis of the stability by HBM}

We will here use the so-called asymtotic numerical method (ANM) continuation technique [9] and a harmonic-based numerical method [10] to determine the stability of the periodic responses of the system.

The stability of two nonlinear normal modes is shown in Fig. 4. in which we can see that the second nonlinear mode becomes instable when $u_{1}(t)$ reaches a maximum point. Fig. 5 show the stability of the periodic forced responses. In Fig. 6, we have ploted a zoom of the periodic responses of $u_{1}(t)$ with stability and the second nonlinear mode with stability. We can see that when the level of forcing is low, the resonance peak of the periodic response follows the second nonlinear mode and there is no SMR. Under increasing the forcing, when the resonance peak of the periodic response arrives at the point from which the the 


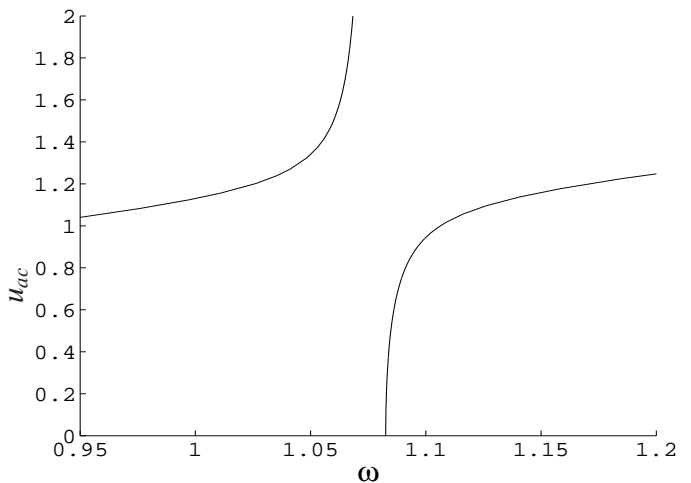

(a)

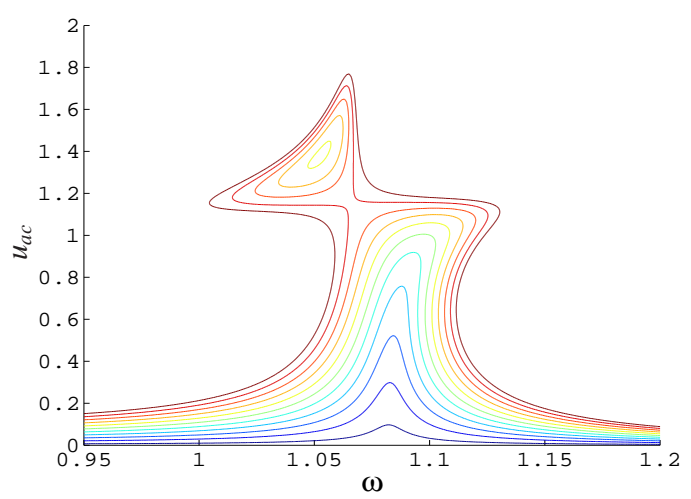

(b)

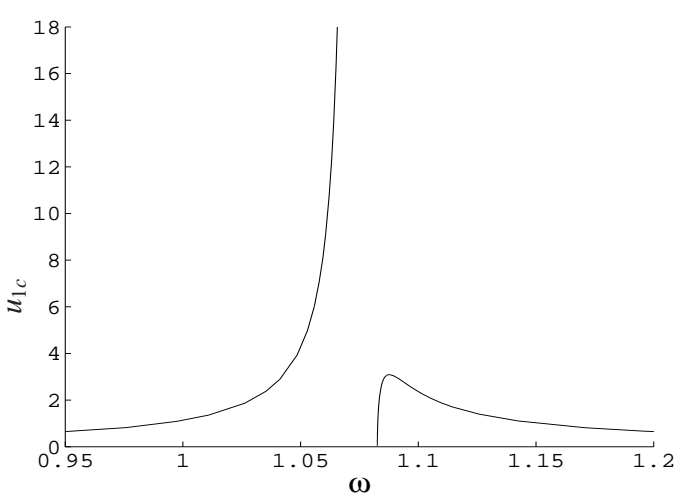

(c)

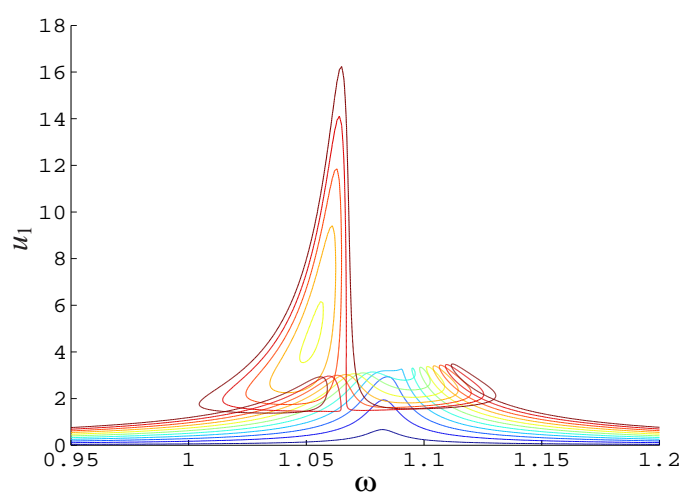

(d)

Fig. 3. Nonlinear normal modes and periodic forced responses. Fig. 3(a) and Fig. 3(c) showing two nonlinear normal modes. Fig. 3 (b) and Fig. 3(d) showing the periodic responses for $F=[0.01$ : $0.02: 0.21]$.

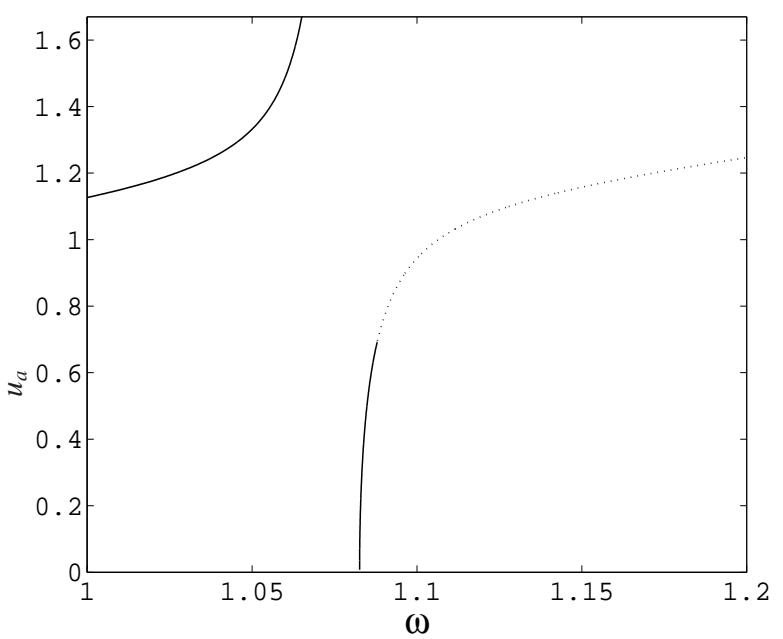

(a)

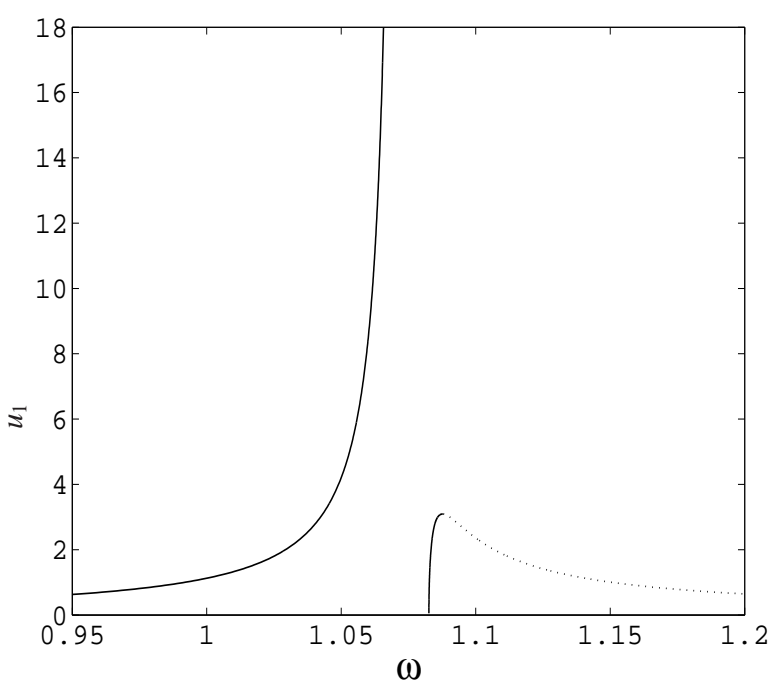

(b)

Fig. 4. The stability of nonlinear normal modes of the system.

second nonlinear mode becomes instable, the periodic responses become instable, SMR begins and the TET phenomenon appears. And the plateau of the resonance peaks corresponds well with the maximum point of the second nonlinear mode. When the force is higher, the additional branches of periodic regimes appear with higher amplitudes.

We have done numerical simulations to illustrate these transitions. The time series are presented for different levels of forcing $F_{1}$ and frequencies of excitation, in Fig. 7. For the frequency of excitation $1.0877 \mathrm{~Hz}$ (Fig. 7(a) and $7(\mathrm{~b})$ ) and $F_{1}=0.03$ (low force), there is no SMR, while for $F_{1}=0.07$, SMR appears. For $F_{1}=0.13$ and a frequency of excitation $1.05 \mathrm{~Hz}$, we can observe the periodic reponses with higher amplitudes that corresponds to the additional branches. 


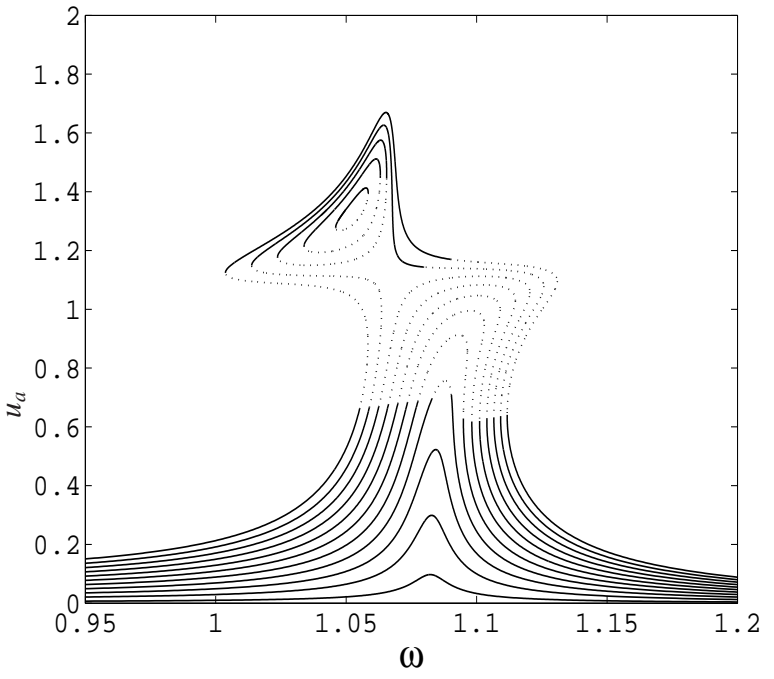

(a)

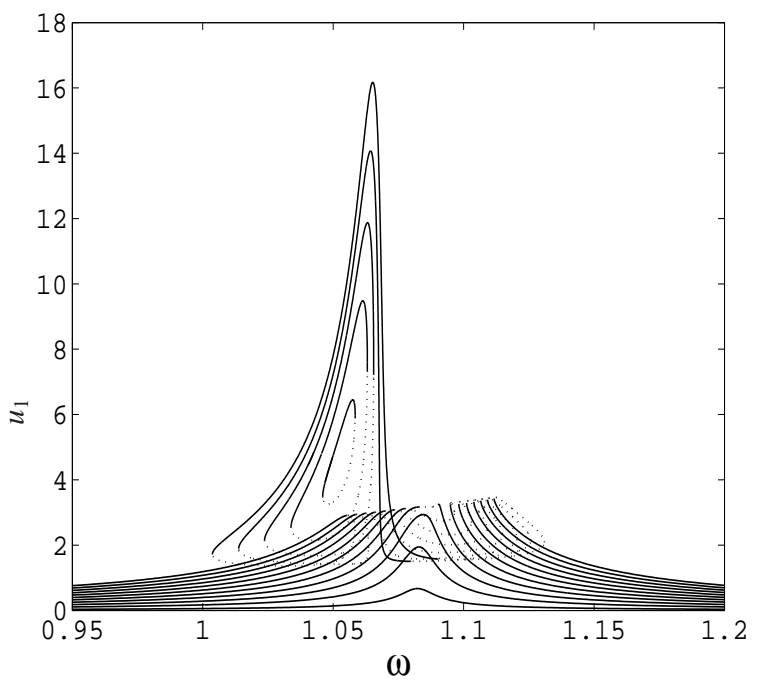

(b)

Fig. 5. The stability of the periodic responses of the system for $F_{1}=[0.01: 0.02: 0.21]$.

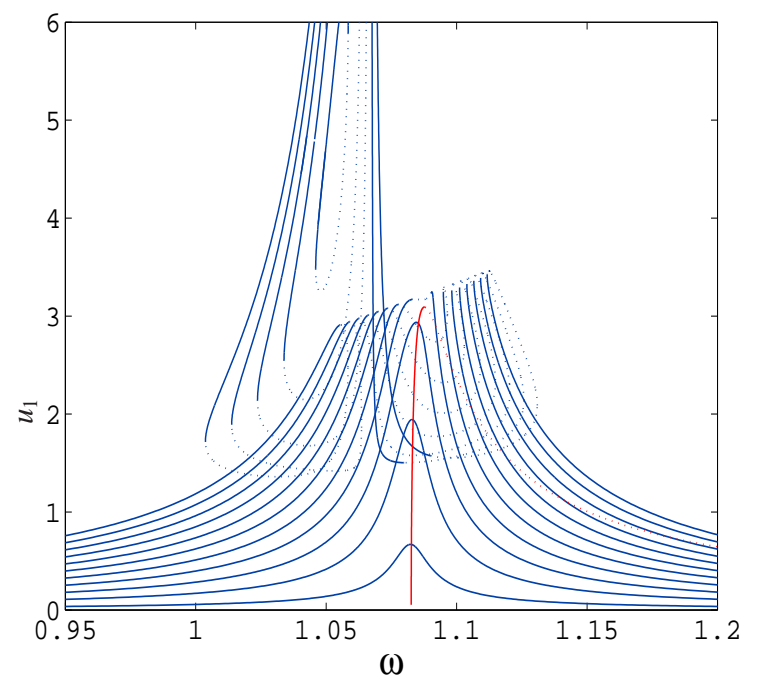

Fig. 6. Zoom onto Fig. 5(b), red curve: the nonlinear mode.

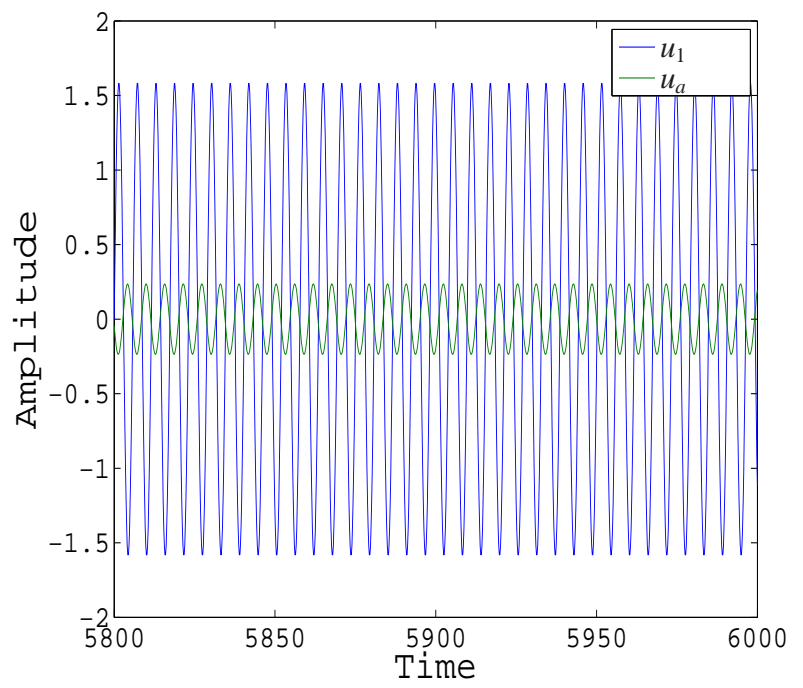

(a) $F_{1}=0.03$

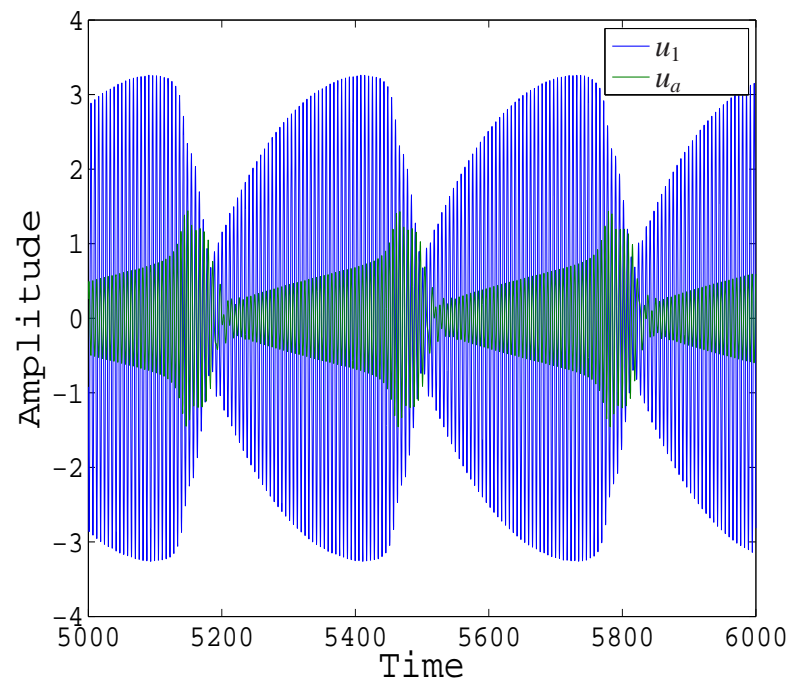

(b) $F_{1}=0.07$

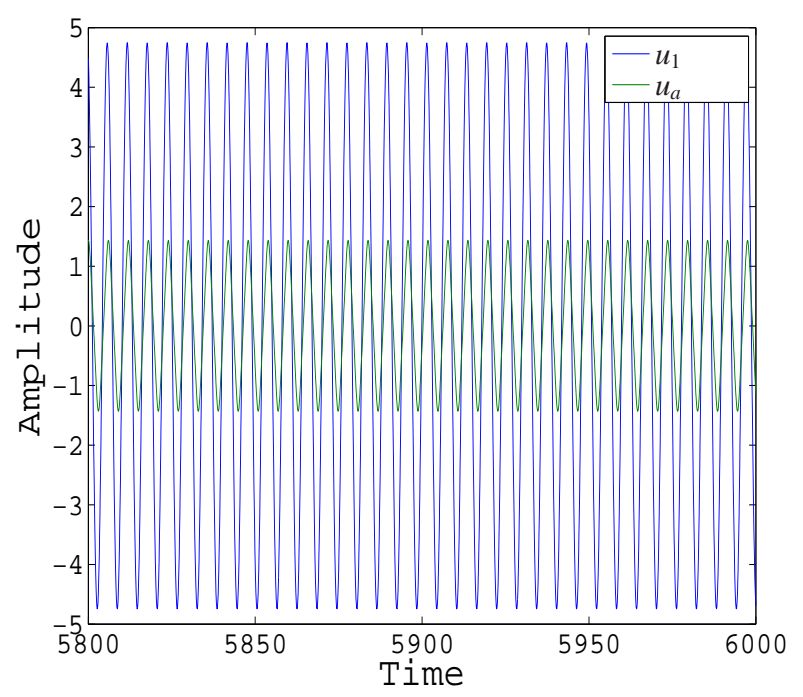

(c) $F_{1}=0.13$

Fig. 7. The time series for three levels of forcing $F_{1} .7(a)$ and 7(b): the frequency of excitation is $1.0877 \mathrm{~Hz}$. 9(a): the frequency of excitation is $1.05 \mathrm{~Hz}$. 


\section{Definition of the desired working zone for the NES}

We have already discussed about the beginning of the TET phenomenon of the system and the appearance of the additional branches of periodic regimes. Here, the two levels of forcing corresponding to the beginning of TET and to the appearance of the additional branches are called "the threshold for the beginning of TET" and "the threshold for the appearance of undesired periodic regimes". The zone of forcing between the two levels, is called "the desired working zone" for the NES. To determine the desired working zone for the NES, we analyze the extrema of the curves of forced responses of $u_{1}(t)$ (Fig. 3(d)) with the constant levels of forcing. The curves of Fig. 8 show the extrema on the plane $\left(\omega, F_{1}\right)$. According to the Fig. 3(d) and Fig. 8, when the level of forcing is low and below the threshold for the beginning of TET for which SMR does not occur, there is only one resonance peak in the periodic solution and it corresponds with one extremum, namely one value of $\omega$. When the level of forcing reaches the threshold for the beginning of TET, the resonance peak arrives at the plateau and begins to deform. At this time, it appears three extrema and three values of $\omega$. When the level of forcing reaches the threshold for the appearance of undesired periodic regimes, the undesired additional branch appears, and henceforth, there are more than three extrema and three values of $\omega$. According to these findings, we determine the desired working zone for NES just like the zone between two lines in Fig. 8 .

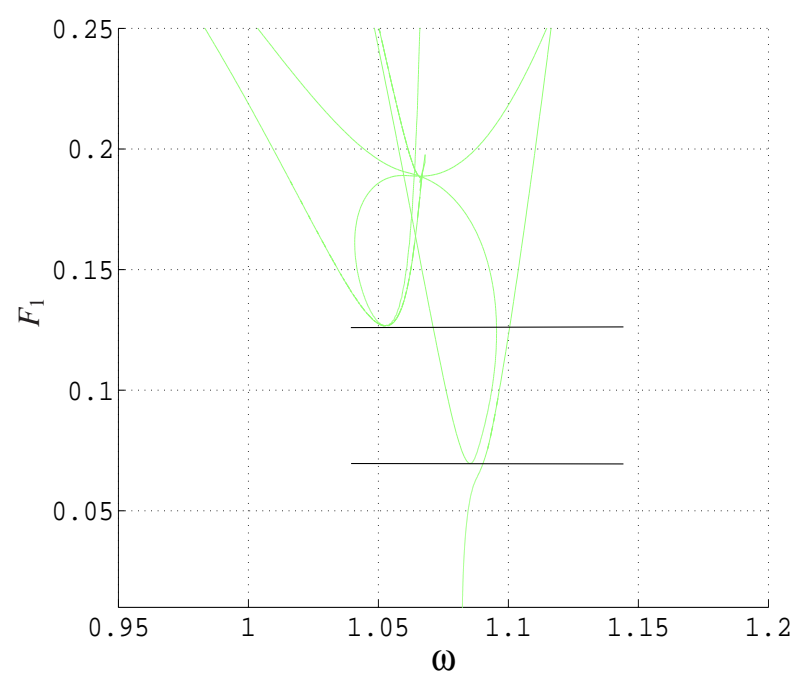

Fig. 8. The desired working zone for the NES.

\subsection{The threshold for the beginning of TET}

The periodic responses of the system with the low and subdivision level of forcing and the second nonlinear mode are shown in Fig. 9. We can see that the resonance peak of the periodic response $u_{1}(t)$ follows well the second nonlinear mode until the maximum point of the second nonlinear mode. And for $u_{a c}(t)$ the resonance peak of the periodic responses also follows well the second nonlinear mode with

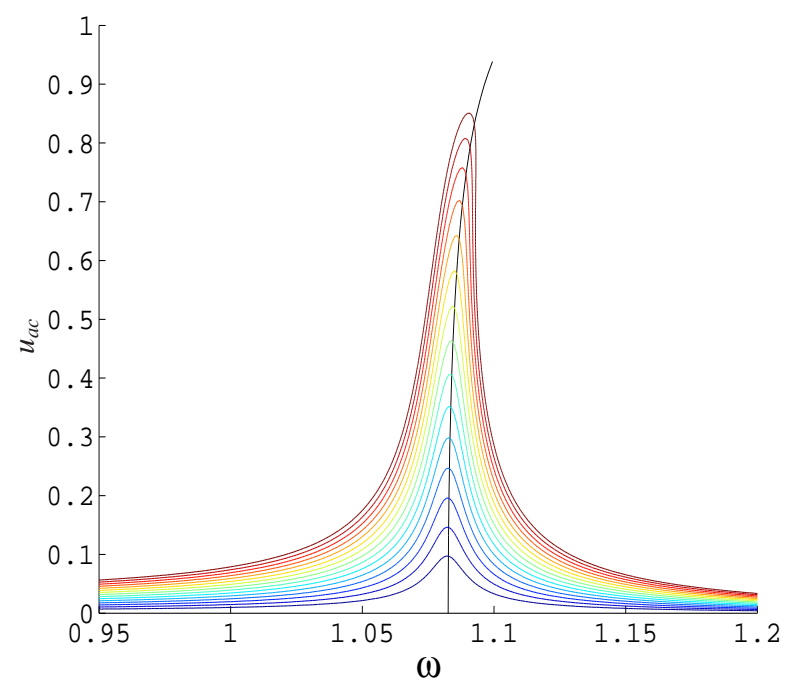

(a)

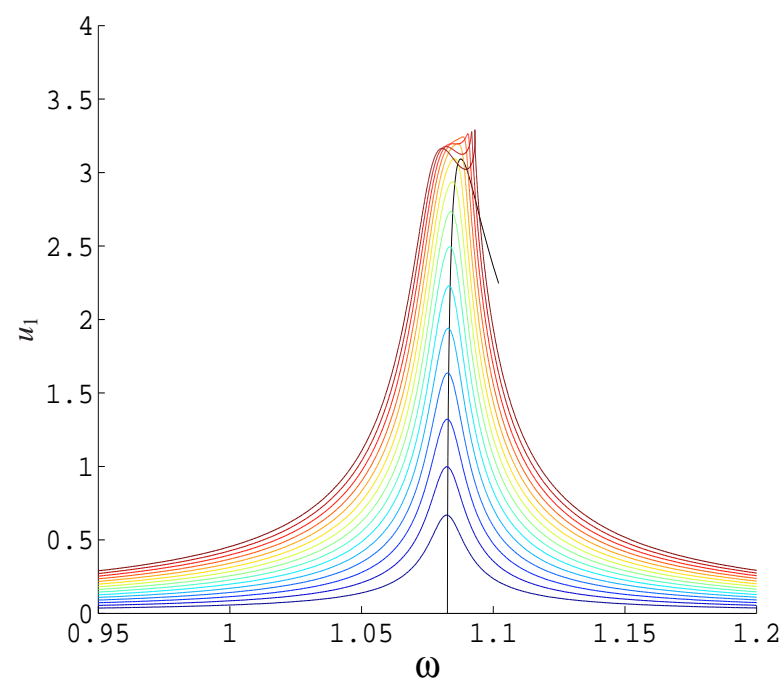

(b)

Fig. 9. The periodic responses with nonlinear normal modes of the system for $F_{1}=[0.01: 0.005: 0.08]$.

certain levels of forcing. Therefore, we will here follow a simplified approach by using the solutions of the maximum point of the second nonlinear mode to determine the threshold for the beginning of TET.

To look for the value of the maximum point of the second nonlinear mode of $u_{1}(t)$, we resolve the equations (2) to express $u_{1 c}$ and $u_{a c}$ according to $\omega$ :

$$
\begin{aligned}
& u_{1 c}(\omega)=\frac{2 \beta}{1+\beta-\omega^{2}} \sqrt{\frac{\beta^{2}+\left(\gamma \omega^{2}-\beta\right)\left(1+\beta-\omega^{2}\right)}{3 \alpha_{a}\left(1+\beta-\omega^{2}\right)}}, \\
& u_{a c}(\omega)=2 \sqrt{\frac{\beta^{2}+\left(\gamma \omega^{2}-\beta\right)\left(1+\beta-\omega^{2}\right)}{3 \alpha_{a}\left(1+\beta-\omega^{2}\right)}}
\end{aligned}
$$

By deriving $u_{1 c}$ over $\omega$, and posing $d u_{1 c} / d \omega=0$, the value of the frequency of the maximum point $\bar{\omega}$ is obtained as follows:

$$
\bar{\omega}=\sqrt{\frac{\beta+\sqrt{\beta^{2}+\gamma^{2}(1+\beta)^{2}-2 \gamma \beta+\gamma \beta^{2}}}{\gamma}}
$$


Then, the values of the two amplitudes of the maximum point $\overline{u_{1 c}}=u_{1 c}(\bar{\omega})$ and $\overline{u_{a c}}=u_{a c}(\bar{\omega})$ are obtained by the equations (4) and (5).

Therefore, we have obtained the solutions of the maximum point of the second nonlinear mode: $\overline{u_{1}}, \overline{u_{a c}}$ and $\bar{\omega}$. For looking for the threshold for the beginning of TET, we use the four algebraic equations (3), in which we have taken the amplitude $u_{a c}$ et the frequency $\omega$ as the master parameters and easily found the solutions for the response surfaces. We will here take $\overline{u_{a c}}$ and $\bar{\omega}$ as the master parameters. Then closed form expressions of the periodic responses $\overline{u_{1 c}}, \overline{u_{1 s}}, \overline{F_{1 c}}$ and $\overline{F_{1 s}}$ are easily obtained according to $\overline{u_{a c}}$ et $\bar{\omega}$

Here, the solution of the maximum point $\overline{u_{1}}$ and the solution of the periodic response $\overline{u_{1}}$ chave the same value based on the second equation of (2) and the third equation of (3). Moreover, $\overline{F_{1 c}}=\mu_{1} \bar{\omega} \bar{u}_{1 s}$ based on the first equation of (2). Finally, based on $F_{1}=\sqrt{F_{1 c}^{2}+F_{1 s}^{2}}$ and $u_{1}=\sqrt{u_{1 c}^{2}+u_{1 s}^{2}}$, the expressions $\overline{u_{1}}$ and $\bar{F}_{1}$ are obtained according to $\overline{u_{a c}}$ et $\bar{\omega}$. We take $F_{b}$ as the threshold for the beginning of TET here, as follows:

$$
F_{b}=\bar{F}_{1}=\sqrt{\left(\mu_{1} \bar{\omega} u_{1 s}\right)^{2}+\left(\left(1+\beta-\bar{\omega}^{2}\right) \overline{u_{1 s}}-\mu_{1} \bar{\omega} \bar{u}_{1 c}\right)^{2}}
$$

For the equation (6), it is an analytical formula of the threshold for the beginning of TET which is obtained by a simple approach. It will be very useful for pratical design of NES.

\section{Conclusion}

In this paper, we have investigated the TET phenomenon under forcing regime by using a simple system with two degrees of freedom. Looking for the stability of the nonlinear normal modes and of the periodic responses of the system, we have explicated the beginning of TET and defined the desired working zone for the NES. An analytical formula of the level of forcing for the beginning of TET was established by a simplified approach. We will now generalize these results to the systems with several degrees of freedom and coupled with several NES. The intended application of this study is to treat the low frequency noise attenuation in acoustic, where no effective disspative mechanism exists.

\section{References}

1. O. Gendelman, L.I. Manevitch, A.F. Vakakis, R.M. Closkey J. Appl. Mecha. 68(1) (2001) 34-42

2. A.F. Vakakis, O. Gendelman J. Appl. Mecha. 68(1) (2001) 42-48

3. D.M. McFarland, L.A. Bergman, A.F. Vakakis Inter. J. Non. Mecha. 40(6) (2005) 891-899

4. B. Cochelin, P. Herzog, P.O. Mattei C.R. Meca. 334 (2006) 639-644

5. R. Bellet, B. Cochelin, P. Herzog, P.O. Mattei J. S. V. 329 (2010) 2768-2791

6. R. Mariani, S. Bellizi, B. Cochelin, P. Herzog, P.O. Mattei J. S. V. 330 (2011) 5245-5258
7. Y. Starosvetsky, O.V. Gendelman Non. Dyna. 64 (2011) $177-195$

8. Nguyen T.A. Thesis of Ecole Centrale de Lyon (2010)

9. B. Cochelin, C. Vergez J. S. V. 324 (2009) 243-262

10. A. Lazarus, O. Thomas C.R. Meca. 338 (2010) 510517 